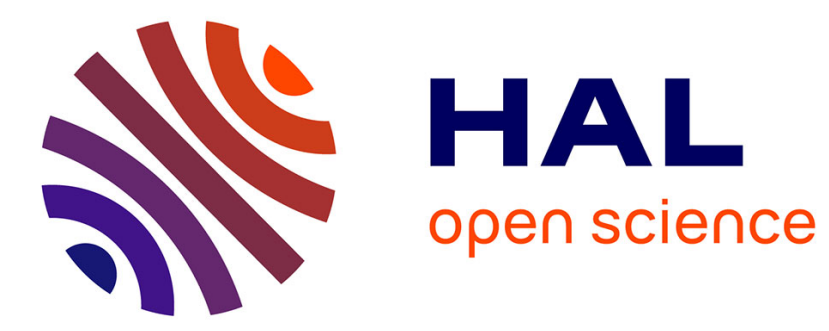

\title{
Central limit theorems for a supercritical branching process in a random environment
}

Hesong Wang, Zhiqiang Gao, Quansheng Liu

\section{To cite this version:}

Hesong Wang, Zhiqiang Gao, Quansheng Liu. Central limit theorems for a supercritical branching process in a random environment. Statistics and Probability Letters, 2011, 81 (5), pp.539-547. 10.1016/j.spl.2011.01.003 . hal-00907157

\section{HAL Id: hal-00907157 https://hal.science/hal-00907157}

Submitted on 21 Nov 2013

HAL is a multi-disciplinary open access archive for the deposit and dissemination of scientific research documents, whether they are published or not. The documents may come from teaching and research institutions in France or abroad, or from public or private research centers.
L'archive ouverte pluridisciplinaire HAL, est destinée au dépôt et à la diffusion de documents scientifiques de niveau recherche, publiés ou non, émanant des établissements d'enseignement et de recherche français ou étrangers, des laboratoires publics ou privés. 


\title{
Central limit theorems for a supercritical branching process in a random environment
}

\author{
Hesong WANG ${ }^{\mathrm{a}, \mathrm{b}}$, Zhiqiang GAO ${ }^{\mathrm{c}, \mathrm{d}, \mathrm{e}}$, Quansheng $\mathrm{LIU}^{\mathrm{b}, \mathrm{d}, \mathrm{e}}$ \\ ${ }^{a}$ College of Mathematics and Computer Science, Hunan Normal University, Changsha, \\ 410076 Hunan, China \\ ${ }^{b}$ College of Mathematics and Computing Science, Changsha University of Science and \\ Technology, Changsha, 410076 Hunan, China \\ ${ }^{c}$ School of Mathematical Sciences, Beijing Normal University, Laboratory of Mathematics \\ and Complex Systems, 100875 Beijing, China \\ ${ }^{d}$ LMAM, Université de Bretagne Sud, Campus de Tohannic, BP 573, 56017 Vannes, France \\ ${ }^{e}$ Université Européenne de Bretagne, France
}

\begin{abstract}
For a supercritical branching process $\left(Z_{n}\right)$ in a stationary and ergodic environment $\xi$, we study the rate of convergence of the normalized population $W_{n}=Z_{n} / E\left[Z_{n} \mid \xi\right]$ to its limit $W_{\infty}$ : we show a central limit theorem for $W_{\infty}-W_{n}$ with suitable normalization and derive a Berry-Esseen bound for the rate of convergence in the central limit theorem when the environment is independent and identically distributed. Similar results are also shown for $W_{n+k}-W_{n}$ for each fixed $k \in \mathbb{N}^{*}$.

Keywords: Branching processes, random environment, central limit theorem, martingale, rate of convergence.

2000 MSC: 60J80, 60F05
\end{abstract}

\section{Introduction}

Galton-Watson processes have been studied by many authors, due to a wide range of applications. See for example the books by Harris (1963) and Athreya and Ney (1972). In a Galton-Watson process $\left\{Z_{n}, n=0,1, \ldots\right\}$, particles behave independently, each gives birth to a random number of particles of the next generation with a fixed distribution $\left\{p_{k}: k=0,1, \ldots\right\}$.

A branching process in a random environment is a natural and important extension of the Galton-Watson process. It is a class of non-homogeneous GaltonWatson processes indexed by a time-environment $\xi=\left(\xi_{0}, \xi_{1}, \xi_{2}, \ldots\right)$, which is

\footnotetext{
Email addresses: hesongwang@tom.com (Hesong WANG), gaozq@bnu.edu.cn (Zhiqiang GAO), quansheng.liu@univ-ubs.fr (Quansheng LIU)

${ }^{1}$ Corresponding author: GAO Zhiqiang, School of Mathematical Sciences, Beijing Normal University, 100875 Beijing, China.
} 
supposed to be stationary and ergodic; given the environment $\xi$, the particles of $n$-th generation have offspring distribution $\left\{p_{k}\left(\xi_{n}\right): k \in \mathbb{N}\right\}$ depending on $\xi_{n}$. For first important works on the subject, see Smith and Wilkinson (1969) and Athreya and Karlin (1971ab).

For a Galton-Waston process with $Z_{0}=1$ and $m=E Z_{1} \in(0, \infty)$, it is well known that $\left\{W_{n}=Z_{n} / m^{n}: n=0,1, \ldots\right\}$ forms a non-negative martingale, and converges almost surely to a random variable $W_{\infty}$. For the convergence rate of the martingale, Heyde (1971) and Bühler (1969) obtained respectively that if $\operatorname{Var}\left(Z_{1}\right)=\sigma^{2}<\infty$, then conditioned on $Z_{n}>0$, the conditional laws of

$$
\left(m^{2}-m\right)^{\frac{1}{2}} \sigma^{-1} Z_{n}^{-\frac{1}{2}} m^{n}\left(W_{\infty}-W_{n}\right)
$$

and

$$
\left(m^{k} /\left(m^{k}-1\right)\right)^{\frac{1}{2}}\left(m^{2}-m\right)^{\frac{1}{2}} \sigma^{-1} Z_{n}{ }^{-\frac{1}{2}} m^{n}\left(W_{n+k}-W_{n}\right) \quad k \in \mathbb{N}^{*}
$$

converge to the normal law $\mathcal{N}(0,1)$; Heyde and Brown (1971) gave an estimation of its convergence rate under a third moment condition.

The object of this paper is to extend the theorems of Bühler (1969), Heyde (1971) and Heyde and Brown (1971) to a branching process in a random environment. The main results are Theorems 2.1 and 2.2 .

\section{Main Results}

As usual, we write $\mathbb{N}=\{0,1,2, \cdots\}, \mathbb{N}^{*}=\{1,2, \cdots\}$ and $\mathbb{R}$ for the set of real numbers.

Let us first recall the definition of a branching process in a random environment. For reference on the subject, see for example Athreya and Karlin (1971a b), and Athreya and Ney (1972).

$A$ random environment $\xi=\left(\xi_{n}\right)$ is formulated as a stationary and ergodic sequence of random variables taking values in some measurable space $(\Theta, \mathcal{F})$. Each realization of $\xi_{n}$ corresponds to a probability distribution $p\left(\xi_{n}\right)=\left\{p_{i}\left(\xi_{n}\right)\right.$ : $i \in \mathbb{N}\}$ where

$$
p_{i}\left(\xi_{n}\right) \geq 0, \quad \sum_{i=0}^{\infty} p_{i}\left(\xi_{n}\right)=1, \quad 0<\sum_{i=0}^{\infty} i p_{i}\left(\xi_{n}\right)<\infty .
$$

Without loss of generality, we can take $\xi_{n}$ as coordinate functions defined on the product space $\left(\Theta^{\mathbb{N}}, \mathcal{F} \otimes \mathbb{N}\right)$, equipped with a probability law $\tau$, which is invariant and ergodic under the usual shift transformation $\theta$ on $\Theta^{\mathbb{N}}: \theta\left(\xi_{0}, \xi_{1}, \cdots\right)=$ $\left(\xi_{1}, \xi_{2}, \cdots\right)$. A branching process $\left(Z_{n}\right)_{n \geq 0}$ in the random environment $\xi$ is a class of non-homogeneous branching processes indexed by $\xi$. By definition,

$$
Z_{0}=1, \quad Z_{n+1}=\sum_{i=1}^{Z_{n}} X_{n, i} \quad n \geq 0
$$


where given $\xi,\left\{X_{n, i}: n \geq 0, i \geq 1\right\}$ is a family of (conditionally) independent random variables, each $X_{n, i}$ has the common law $p\left(\xi_{n}\right)$. Notice that when all $\xi_{n}$ are the same constant, $\left(Z_{n}\right)$ reduces to the classical Galton-Waston process.

Let $\left(\Gamma, P_{\xi}\right)$ be the probability space under which the process is defined when the environment $\xi$ is given. As usual, $P_{\xi}$ is called quenched law. The total probability space can be formulated as the product space $\left(\Gamma \times \Theta^{\mathbb{N}}, P\right)$, where $P=P_{\xi} \otimes \tau$ in the sense that for all measurable and positive function $g$, we have

$$
\int g d P=\iint g(\xi, y) d P_{\xi}(y) d \tau(\xi)
$$

(recall that $\tau$ is the law of the environment $\xi$ ). The total probability $P$ is usually called annealed law. The quenched law $P_{\xi}$ may be considered to be the conditional probability of the annealed law $P$ given $\xi$. The expectation with respect to $P_{\xi}$ (resp. $P$ ) will be denoted $E_{\xi}$ (resp. $E$ ).

For $n \geq 0$, define

$$
\begin{aligned}
& m_{n}(a)=m\left(\xi_{n}, a\right)=\sum_{i=1}^{\infty} i^{a} p_{i}\left(\xi_{n}\right), \quad a \in \mathbb{R}, \\
& m_{n}=m_{n}(1), \quad \sigma_{n}^{2}=m_{n}(2)-m_{n}^{2}, \\
& \pi_{0}=1 \quad \text { and } \quad \pi_{n}=\pi_{n}(\xi)=m_{0} \cdots m_{n-1} \quad \text { for } \quad n \geq 1 .
\end{aligned}
$$

Then $\pi_{n}=E_{\xi} Z_{n}$ for $n \geq 0$. It is well known that

$$
W_{n}=Z_{n} / \pi_{n}
$$

is a martingale with respect to the filtration

$$
\mathcal{F}_{0}=\{\varnothing, \Omega\}, \quad \mathcal{F}_{n}=\sigma\left\{\xi, X_{j, i}: j \leq n-1, i \geq 1\right\} \quad(n \geq 1),
$$

so that the limit

$$
W_{\infty}=\lim _{n \rightarrow \infty} W_{n}
$$

exists almost surely (a.s.) with $E W \leq 1$ by Fatou's lemma.

Throughout the paper, we always assume that

$$
E \ln m_{0}>0 \quad \text { and } \quad E\left(\frac{Z_{1}}{m_{0}} \ln ^{+} Z_{1}\right)<\infty .
$$

The first assumption ensures that the process is supercritical (cf. Athreya and Karlin (1971a)); the second one together with the first implies that $E W_{\infty}=1$; moreover,

$$
P_{\xi}\left(W_{\infty}>0\right)=P_{\xi}\left(Z_{n} \rightarrow \infty\right)=\lim _{n \rightarrow \infty} P_{\xi}\left(Z_{n}>0\right)=1-q(\xi)>0 \quad \text { a.s. }
$$

where $q(\xi)=\lim _{n \rightarrow \infty} P_{\xi}\left(Z_{n}=0\right)$ is the extinct probability. 
In this paper, we search for central limit theorems on $W_{\infty}-W_{n}$ and $W_{n+k}-$ $W_{n}$ for fixed $k \geq 1$ with an appropriate normalization. Assum that $m_{0}(2)<\infty$ a.s., and let

$$
\Delta_{k}^{2}=\Delta_{k}^{2}(\xi)=\sum_{0 \leq i<k} \frac{1}{\pi_{i}} \frac{\sigma_{i}^{2}}{m_{i}^{2}} \quad \text { for } \quad k \in \mathbb{N}^{*} \cup\{\infty\} .
$$

Then for $k \in \mathbb{N}^{*}, \Delta_{k}^{2}(\xi)$ is the variance of $W_{k}$ under $P_{\xi} ; \Delta_{\infty}^{2}(\xi)$ is the variance of $W_{\infty}$ if the series converges (i.e. $\Delta_{\infty}^{2}(\xi)<\infty$ ): see Lemma 3.2 .

We can now formulate our first main result.

Theorem 2.1. Suppose that (10) holds and that $m_{0}(2)<\infty$ a.s.. In the case where $k=\infty$, assume additionally that $E \ln ^{+}\left(\sigma_{0}^{2} / m_{0}^{2}\right)<\infty$. Write

$$
U_{n, k}=\frac{\pi_{n}\left(W_{n+k}-W_{n}\right)}{\sqrt{Z_{n}} \Delta_{k}\left(\theta^{n} \xi\right)} \quad \text { for } \quad k \in \mathbb{N}^{*} \cup\{\infty\},
$$

where by convention $W_{n+k}=W_{\infty}$ if $k=\infty$. Then for each $k \in \mathbb{N}^{*} \cup\{\infty\}$, as $n \rightarrow \infty$,

$$
\sup _{x \in \mathbb{R}}\left|P_{\xi}\left(U_{n, k} \leq x \mid Z_{n}>0\right)-\Phi(x)\right| \rightarrow 0 \quad \text { in } \quad L^{1},
$$

and

$$
\sup _{x \in \mathbb{R}}\left|P\left(U_{n, k} \leq x \mid Z_{n}>0\right)-\Phi(x)\right| \rightarrow 0 .
$$

We believe that for each $k \in \mathbb{N} \cup\{\infty\}$

$$
\lim _{n \rightarrow \infty} \sup _{x \in \mathbb{R}}\left|P_{\xi}\left(U_{n, k} \leq x \mid Z_{n}>0\right)-\Phi(x)\right|=0 \quad \text { a.s.. }
$$

We notice that in the classical Galton-Waston process, 13 reduces to the results of Bühler (1969) and Heyde (1971). Our second main result concerns the rate of convergence in the above central limit theorem for a branching process with an independent and identically distributed environment.

Theorem 2.2. Let the environment $\left\{\xi_{n}\right\}$ be independent and identically distributed. Assume that (10) holds and that $m_{0}(2)<\infty$ a.s.. In the case where $k=\infty$, assume additionally that $E \ln ^{+}\left(\sigma_{0}^{2} / m_{0}^{2}\right)<\infty$. For each $k \in \mathbb{N}^{*} \cup\{\infty\}$, if $E\left|\frac{W_{k}-1}{\Delta_{k}}\right|^{2+\delta}<\infty$ for some $\delta \in(0,1]$, then

$$
\sup _{x \in \mathbb{R}}\left|P\left(U_{n, k} \leq x \mid Z_{n}>0\right)-\Phi(x)\right| \leq \frac{C_{\delta}\left(E m_{0}\left(-\frac{\delta}{2}\right)\right)^{n} E\left|\frac{W_{k}-1}{\Delta_{k}}\right|^{2+\delta}}{P\left(Z_{n}>0\right)},
$$

where $U_{n, k}$ is defined in Theorem 2.1 and $C_{\delta}$ is the Berry-Esseen constant.

Remark 2.3. It maybe useful to notice that if

$$
E\left(Z_{1} / m_{0}\right)^{2+\delta}<\infty, \quad E m_{0}^{-(1+\delta)}<1 \quad \text { and } \quad m_{0}(2) / m_{0}^{2} \geq A
$$


for some constant $A>1$, then $E\left|\frac{W_{\infty}-1}{\Delta_{\infty}}\right|^{2+\delta}<+\infty$. In fact by Theorem 3 of Guivarc'h and Liu (2001), the first two conditions imply that $E\left|W_{\infty}-1\right|^{2+\delta}<$ $\infty$, while the last one implies that $\Delta_{\infty}^{2} \geq A-1>0$.

For the classical Galton-Watson process with $\delta=1$, Theorem 2.2 reduces to Theorem 2 of (Heyde and Brown, 1971, p.272).

\section{Proof of Theorem 2.1}

In this section, we consider a central limit theorem under a second moment condition in proving Theorem 2.1. We first give some lemmas.

Lemma 3.1 (Grincevičjus (1974)). Let $\left\{\left(\alpha_{n}, \beta_{n}\right), n=0,1,2, \cdots\right\}$ be a stationary and ergodic sequence of random variables with values in $\mathbb{R}^{2}$. If

$$
E \ln \left|\alpha_{0}\right|<0 \text { and } E \ln ^{+}\left|\beta_{0}\right|<\infty,
$$

then

$$
\sum_{n=0}^{\infty}\left|\alpha_{0} \alpha_{1} \cdots \alpha_{n-1} \beta_{n}\right|<\infty \quad \text { a.s. }
$$

In fact, the result is a direct consequence of the ergodic theorem and Cauchy's criterion for the convergence of series.

Using the above lemma, we can easily obtain the following result.

Lemma 3.2. Under the assumptions in Theorem 2.1, for each $k \in \mathbb{N}^{*} \cup\{\infty\}$,

$$
\operatorname{Var}_{\xi}\left(W_{k}\right)=\Delta_{k}^{2}(\xi)=\sum_{0 \leq i<k} \frac{1}{\pi_{i}} \frac{\sigma_{i}^{2}}{m_{i}^{2}}
$$

This has been known for branching processes in varying environment, see e.g. (Jagers, 1974, p.175) in a slightly different form. For reader's convenience, we present a proof in the following.

Proof of Lemma 3.2 By (2) and the definition of $W_{n}$, we have

$$
W_{n+1}-W_{n}=\frac{1}{\pi_{n}} \sum_{j=1}^{Z_{n}}\left(\frac{X_{n, j}}{m_{n}}-1\right) .
$$

Recall that under $P_{\xi}$, the random variables $\left\{X_{n, j}\right\}$ are independent of each other and have the common distribution $p\left(\xi_{n}\right)$ with expectation $m_{n}$. Hence a direct calculation shows that

$$
\begin{aligned}
E_{\xi}\left(\left(W_{n+1}-W_{n}\right)^{2}\right) & =E_{\xi}\left(E_{\xi}\left(\left(W_{n+1}-W_{n}\right)^{2} \mid \mathcal{F}_{n}\right)\right) \\
& =E_{\xi}\left(\frac{Z_{n}}{\pi_{n}^{2}} \frac{\sigma_{n}^{2}}{m_{n}^{2}}\right)=\frac{1}{\pi_{n}} \frac{\sigma_{n}^{2}}{m_{n}^{2}} .
\end{aligned}
$$


As $\left\{W_{n}\right\}$ is a martingale, it follows that

$$
E_{\xi} W_{k}^{2}=E_{\xi} W_{0}^{2}+\sum_{i=0}^{k-1} E\left(\left(W_{i+1}-W_{i}\right)^{2}\right)=1+\sum_{i=0}^{k-1} \frac{1}{\pi_{i}} \frac{\sigma_{i}^{2}}{m_{i}^{2}} .
$$

Therefore for each fixed integer $k$,

$$
\operatorname{Var}_{\xi}\left(W_{k}\right)=E_{\xi}\left(W_{k}^{2}\right)-1=\sum_{i=0}^{k-1} \frac{1}{\pi_{i}} \frac{\sigma_{i}^{2}}{m_{i}^{2}} .
$$

Now we turn to the calculation of $\operatorname{Var}_{\xi}\left(W_{\infty}\right)$. By Lemma 3.1. when $E \ln m_{0}>0$ and $E \ln ^{+} \frac{\sigma_{i}^{2}}{m_{i}^{2}}<\infty$,

$$
\sup _{n} E_{\xi}\left(W_{n}^{2}\right)=1+\sum_{i=0}^{\infty} \frac{1}{\pi_{i}} \frac{\sigma_{i}^{2}}{m_{i}^{2}}<\infty \quad \text { a.s. }
$$

So $W_{n}$ converges to $W_{\infty}$ in $L^{2}$ under $P_{\xi}$ and

$$
E_{\xi}\left(W_{\infty}^{2}\right)=\lim _{k \rightarrow \infty} E_{\xi}\left(W_{k}^{2}\right)=1+\sum_{i=0}^{\infty} \frac{1}{\pi_{i}} \frac{\sigma_{i}^{2}}{m_{i}^{2}}
$$

It follows that

$$
\operatorname{Var}_{\xi}\left(W_{\infty}\right)=E_{\xi}\left(W_{\infty}^{2}\right)-1=\Delta_{\infty}^{2}(\xi)=\sum_{i=0}^{\infty} \frac{1}{\pi_{i}} \frac{\sigma_{i}^{2}}{m_{i}^{2}}<\infty \quad \text { a.s. }
$$

To give our next lemma, we will need some notations, which will also be used in the proof of the main theorems. By definition,

$$
Z_{n+k}=\sum_{j=1}^{Z_{n}} Z_{k}(n, j)
$$

where $Z_{k}(n, j)$ denotes the number of descendants in the $(n+k)$-th generation of the $j$-th particle among the $Z_{n}$ particles in $n$-th generation.

Writing $W_{k}(n, j)=\frac{Z_{k}(n, j)}{\pi_{k}\left(\theta^{n} \xi\right)}$ and using 16 , we obtain the following decomposition:

$$
\pi_{n}\left(W_{n+k}-W_{n}\right)=\sum_{j=1}^{Z_{n}}\left(W_{k}(n, j)-1\right) .
$$

Letting $k \rightarrow \infty$, it follows that

$$
\pi_{n}\left(W_{\infty}-W_{n}\right)=\sum_{j=1}^{Z_{n}}\left(W_{\infty}(n, j)-1\right),
$$


where under $P_{\xi}$, the random variables $\left\{W_{\infty}(n, j)\right\}_{j}$ are independent of each other and have the common conditional distribution

$$
P_{\xi}\left(W_{\infty}(n, j) \in \cdot\right)=P_{\theta^{n} \xi}\left(W_{\infty} \in \cdot\right) .
$$

Lemma 3.3. Suppose that the assumptions of Theorem 2.1 hold. Let $r_{n} \in \mathbb{N}$ with $r_{n} \rightarrow \infty$. For $k \in \mathbb{N}^{*} \cup\{\infty\}$, define

$$
Y_{k, n}=\frac{1}{\sqrt{r_{n}}} \sum_{j=1}^{r_{n}} \frac{W_{k}(n, j)-1}{\Delta_{k}\left(\theta^{n} \xi\right)} .
$$

Fix $k \in \mathbb{N}^{*} \cup\{\infty\}$. Then for each subsequence $\left\{n^{\prime}\right\}$ of $\mathbb{N}$ with $n^{\prime} \rightarrow \infty$, there is a subsequence $\left\{n^{\prime \prime}\right\}$ of $\left\{n^{\prime}\right\}$ with $n^{\prime \prime} \rightarrow \infty$ such that for a.e. $\xi$ and all $x \in \mathbb{R}$, as $n^{\prime \prime} \rightarrow \infty$,

$$
P_{\xi}\left(Y_{k, n^{\prime \prime}} \leq x\right) \rightarrow \Phi(x) .
$$

Proof. Fix $k \in \mathbb{N}^{*} \cup\{\infty\}$. In order to use Lindeberg's theorem, for $n \in \mathbb{N}$ and $\epsilon>0$, we consider the quantity

$$
L_{k}(\xi, \epsilon, n)=\frac{1}{r_{n}} \sum_{j=1}^{r_{n}} E_{\xi}\left(\left(\frac{W_{k}(n, j)-1}{\Delta_{k}\left(\theta^{n} \xi\right)}\right)^{2} ;\left|\frac{W_{k}(n, j)-1}{\Delta_{k}\left(\theta^{n} \xi\right) \sqrt{r_{n}}}\right|>\epsilon\right),
$$

where for a set $A$, we write $E_{\xi}(x ; A)$ for $E_{\xi}\left(X \mathbf{1}_{A}\right), \mathbf{1}_{A}$ denoting the indicator function of $A$. By the stationarity and ergodicity of the environment, for all $\epsilon>0$, as $n \rightarrow \infty$,

$$
E L_{k}(\xi, \epsilon, n)=E\left[\left(\frac{W_{k}-1}{\Delta_{k}}\right)^{2} ;\left|\frac{W_{k}-1}{\Delta_{k}}\right|>\sqrt{r_{n}} \epsilon\right] \rightarrow 0 .
$$

Let $\left\{n^{\prime}\right\}$ be a subsequence of $\mathbb{N}$. Notice that from 19 , we can choose a subsequence $\left\{n^{\prime \prime}\right\}$ for which $L_{k}\left(\xi, \varepsilon, n^{\prime \prime}\right) \rightarrow 0$ a.s., but this sequence may depend of $\epsilon$. We will use a diagonal argument to select a subsequence $\left\{n^{\prime \prime}\right\}$ of $\left\{n^{\prime}\right\}$ such that a.s. $L_{k}\left(\xi, \varepsilon, n^{\prime \prime}\right) \stackrel{n^{\prime \prime} \rightarrow \infty}{\longrightarrow} 0$ for all $\epsilon>0$. Set

$$
\epsilon_{m}=1 / m \text { for } m \geq 1 \text {. }
$$

Let $\left\{n_{0, i}\right\}=\left\{n^{\prime}\right\}$. Because of $\sqrt{19}$, there is a subsequence $\left\{n_{1, i}\right\}$ of $\left\{n_{0, i}\right\}$ and a set $\Lambda_{1}$ with $\tau\left(\Lambda_{1}\right)=1$ such that $\forall \xi \in \Lambda_{1}$,

$$
\lim _{i \rightarrow \infty} L_{k}\left(\xi, \epsilon_{1}, n_{1, i}\right)=0 .
$$

Inductively for $m \geq 1$, when $\Lambda_{m}$ and $\left\{n_{m, i}\right\}$ are defined such that $\tau\left(\Lambda_{m}\right)=1$ and $\forall \xi \in \Lambda_{m}, L_{k}\left(\xi, \epsilon_{m}, n_{m, i}\right) \rightarrow 0$, there is a subsequence $\left\{n_{m+1, i}\right\} \subset\left\{n_{m, i}\right\}$ and a set $\Lambda_{m+1}$ with $\tau\left(\Lambda_{m+1}\right)=1$ such that $\forall \xi \in \Lambda_{m+1}$,

$$
\lim _{i \rightarrow \infty} L_{k}\left(\xi, \epsilon_{m+1}, n_{m+1, i}\right)=0 .
$$


We now consider the diagonal sequence $\left\{n_{i . i}\right\}_{i \geq 1}$ and $\Lambda=\bigcap_{j=1}^{\infty} \Lambda_{j}$. For each fixed $\epsilon>0$, let $m \geq \frac{1}{\epsilon}$. Then $\epsilon_{m} \leq \epsilon$ and by the monoticity of $L_{k}(\xi, \epsilon, n)$ in $\epsilon$, we see that $\forall \xi \in \Lambda$,

$$
L_{k}\left(\xi, \epsilon, n_{m, i}\right) \leq L_{k}\left(\xi, \epsilon_{m}, n_{m, i}\right) \rightarrow 0 \quad \text { as } \quad i \rightarrow \infty .
$$

As $\left\{n_{i, i}\right\}$ is a subsequence of $\left\{n_{m, i}\right\}$ whenever $i>m$, this implies that

$$
\lim _{i \rightarrow \infty} L_{k}\left(\xi, \epsilon, n_{i, i}\right)=0 .
$$

Since $\tau(\Lambda)=1$, we have shown that for all $\epsilon>0,20$ holds a.s.. It follows that a.s. 20 holds for all rational $\epsilon>0$, and therefore for all real $\epsilon>0$ by the monoticity of $L_{k}\left(\xi, \epsilon, n_{i i}\right)$ in $\epsilon$. So by Lindeberg's theorem, it is a.s. that for all $x \in \mathbb{R}$, as $i \rightarrow \infty$,

$$
P_{\xi}\left(Y_{k, n_{i, i}} \leq x\right) \rightarrow \Phi(x) .
$$

Thus the lemma has been proved with $\left\{n^{\prime \prime}\right\}=\left\{n_{i, i}\right\}$.

Proof of Theorem 2.1. We shall only deal with the case where $k=\infty$, as the case where $k \in \mathbb{N}^{*}$ can be treated similarly.

We first prove the following assertion: for each sequence $\left\{n^{\prime}\right\}$ of $\mathbb{N}$ with $n^{\prime} \rightarrow \infty$, there exist a subsequence $\left\{n^{\prime \prime}\right\}$ of $\left\{n^{\prime}\right\}$ with $n^{\prime \prime} \rightarrow \infty$ such that for a.e. $\xi$ and all $x$, as $n^{\prime \prime} \rightarrow \infty$,

$$
P_{\xi}\left(U_{n^{\prime \prime}, \infty} \leq x \mid Z_{n^{\prime \prime}}>0\right) \rightarrow \Phi(x) .
$$

By the definition of $U_{n, \infty}$ and the relation $(18)$, we get :

$$
U_{n, \infty}=\frac{\pi_{n}\left(W_{\infty}-W_{n}\right)}{\sqrt{Z_{n}} \Delta_{\infty}\left(\theta^{n} \xi\right)}=\frac{1}{\sqrt{Z_{n}}} \sum_{j=1}^{Z_{n}} \frac{W_{\infty}(n, j)-1}{\Delta_{\infty}\left(\theta^{n} \xi\right)}
$$

where we recall that under $P_{\xi},\left\{W_{\infty}(n, j), j \geq 1\right\}$ is a family of random variables independent of each other and independent of $Z_{n}$, each has the same law as $W_{\infty}$ under $P_{\theta^{n}} \xi$. Set

$$
u_{n}(r, x)=P_{\xi}\left(\frac{1}{\sqrt{r}} \sum_{j=1}^{r} \frac{W_{\infty}(n, j)-1}{\Delta_{\infty}\left(\theta^{n} \xi\right)} \leq x\right), \quad r \in \mathbb{N}^{*}, \quad x \in \mathbb{R} .
$$

Then

$$
\begin{aligned}
P_{\xi}\left(U_{n, \infty} \leq x \mid Z_{n}>0\right) & =\left[P_{\xi}\left(Z_{n}>0\right)\right]^{-1} \sum_{r=1}^{\infty} P_{\xi}\left(U_{n, \infty} \leq x, Z_{n}=r\right) \\
& =\sum_{r=1}^{\infty} u_{n}(r, x) \frac{P_{\xi}\left(Z_{n}=r\right)}{P_{\xi}\left(Z_{n}>0\right)}
\end{aligned}
$$


To show the main idea, let us first consider the special case where $q(\xi)=0$ a.s., i.e. for a.e. $\xi$,

$$
Z_{n} \rightarrow \infty \quad P_{\xi}^{*} \text {-a.s.. }
$$

In this case, the relation 22 becomes

$$
P_{\xi}\left(U_{n, \infty} \leq x\right)=\sum_{r=1}^{\infty} u_{n}(r, x) P_{\xi}\left(Z_{n}=r\right)=E_{\xi} u_{n}\left(Z_{n}, x\right) .
$$

By Lemma 3.3. for each subsequence $\left\{n^{\prime}\right\}$ of $\mathbb{N}$ with $n^{\prime} \rightarrow \infty$, there exist a subsequence $\left\{n^{\prime \prime}\right\}$ of $\left\{n^{\prime}\right\}$ with $n^{\prime \prime} \rightarrow \infty$ such that for a.e. $\xi$ and all $x$, as $n^{\prime \prime} \rightarrow \infty$,

$$
u_{n^{\prime \prime}}\left(Z_{n^{\prime \prime}}, x\right) \rightarrow \Phi(x) .
$$

By the dominated convergence theorem, for a.e. $\xi$ and all $x$, as $n^{\prime \prime} \rightarrow \infty$,

$$
P_{\xi}\left(U_{n^{\prime \prime}, \infty} \leq x\right)=E_{\xi}\left[u_{n^{\prime \prime}}\left(Z_{n^{\prime \prime}}, x\right)\right] \rightarrow \Phi(x) .
$$

So we have proved (21) when $q(\xi)=0$ a.s..

We now consider the general case where $0 \leq q(\xi)<1$ a.s..

For each $\xi \in \Theta^{\mathbb{N}}$, let $Z_{n}^{*}$ be random variables defined on some probability space $\left(\Gamma^{*}, \mathbb{P}_{\xi}^{*}\right)$ with law

$$
P_{\xi}^{*}\left(Z_{n}^{*}=r\right)=\frac{P_{\xi}\left(Z_{n}=r\right)}{P_{\xi}\left(Z_{n}>0\right)}, \quad r \in \mathbb{N}^{*}
$$

Then

$$
P_{\xi}\left(U_{n, \infty} \leq x \mid Z_{n}>0\right)=E_{\xi}^{*} u_{n}\left(Z_{n}^{*}, x\right),
$$

where $E_{\xi}^{*}$ denotes the expectation with respect to $P_{\xi}^{*}$.

Let $\left\{n^{\prime}\right\}$ be a sequence of $\mathbb{N}$ with $n^{\prime} \rightarrow \infty$. If for a.e. $\xi$,

$$
Z_{n^{\prime}}^{*} \rightarrow \infty \quad P_{\xi}^{*} \text {-a.s. }
$$

then as above we can use Lemma 3.3 and the dominated convergence theorem to show that there is a sequence $\left\{n^{\prime \prime}\right\}$ of $\left\{n^{\prime}\right\}$ with $n^{\prime \prime} \rightarrow \infty$ such that for all $x$, as $n^{\prime \prime} \rightarrow \infty$,

$$
E_{\xi}^{*} u_{n^{\prime \prime}}\left(Z_{n^{\prime \prime}}^{*}, x\right) \rightarrow \Phi(x) .
$$

By the fact that $Z_{n}^{*} \rightarrow \infty$ in probability under $P_{\xi}^{*}$, we can choose a subsequence for which $Z_{n}^{*} \rightarrow \infty P_{\xi}^{*}$-a.s.. But to apply Lemma 3.3 . we need that the sequence does not depend on $\xi$. We therefore pass to the probability $P^{*}$ to overcome this difficulty, where $P^{*}=P_{\xi}^{*} \otimes \tau$ is defined on the product space $\Gamma^{*} \times \Theta^{\mathbb{N}}$ just as $P$ was defined on $\Gamma \times \Theta^{\mathbb{N}}$.

Notice that for each $r \in \mathbb{N}^{*}$, as $n \rightarrow \infty$,

$$
P_{\xi}^{*}\left(Z_{n}^{*}=r\right)=\frac{P_{\xi}\left(Z_{n}=r\right)}{P_{\xi}\left(Z_{n}>0\right)} \rightarrow 0,
$$


where the last step holds as $Z_{n} \rightarrow \infty$ a.s. on the survival event $S=\left\{Z_{n}>\right.$ $0, \forall n \geq 1\}$ (see (11) or Tanny (1977) for this fact). Then $Z_{n}^{*} \rightarrow+\infty$ in probability under $P_{\xi}^{*}$. By the dominated convergence theorem, this implies that $Z_{n}^{*} \rightarrow+\infty$ in probability under $P^{*}$. Therefore for each subsequence $\left\{n^{\prime}\right\}$ of $\mathbb{N}$ with $n^{\prime} \rightarrow \infty$, there is a subsequence $\{\tilde{n}\} \subset\left\{n^{\prime}\right\}$ with $\tilde{n} \rightarrow \infty$ such that $Z_{\tilde{n}}^{*} \rightarrow+\infty$ a.s. under $P^{*}$. This implies that for a.e. $\xi$, as $\tilde{n} \rightarrow \infty$,

$$
Z_{\tilde{n}}^{*} \rightarrow+\infty \quad P_{\xi}^{*} \text {-a.s. }
$$

Now by Lemma 3.3 , there exists a subsequence $\left\{n^{\prime \prime}\right\}$ of $\{\tilde{n}\}$ such that for a.e. $\xi$ and all $x$, as $n^{\prime \prime} \rightarrow \infty$,

$$
u_{n^{\prime \prime}}\left(Z_{n^{\prime \prime}}^{*}, x\right) \rightarrow \Phi(x)
$$

By the dominated convergence theorem, for almost every $\xi$ and each $x$, as $n^{\prime \prime} \rightarrow \infty$,

$$
P_{\xi}\left(U_{n^{\prime \prime}, \infty} \leq x \mid Z_{n^{\prime \prime}}>0\right)=E_{\xi} u_{n^{\prime \prime}}\left(Z_{n^{\prime \prime}}^{*}, x\right) \rightarrow \Phi(x) .
$$

So combining the above two cases, we have proved (21).

Since $P_{\xi}\left(U_{n^{\prime \prime}, \infty} \leq x \mid Z_{n^{\prime \prime}}>0\right)$ are distribution functions and $\Phi(x)$ is a continuous distribution function, by Dini's Theorem we see that for a.e. $\xi$, as $n^{\prime \prime} \rightarrow \infty$,

$$
\sup _{x}\left|P_{\xi}\left(U_{n^{\prime \prime}, \infty} \leq x \mid Z_{n^{\prime \prime}}>0\right)-\Phi(x)\right| \rightarrow 0 .
$$

By the dominated convergence theorem, 24 implies that as $n^{\prime \prime} \rightarrow \infty$,

$$
E \sup _{x}\left|P_{\xi}\left(U_{n^{\prime \prime}, \infty} \leq x \mid Z_{n^{\prime \prime}}>0\right)-\Phi(x)\right| \rightarrow 0 \text {. }
$$

Therefore we have proved that for each sequence $\left\{n^{\prime}\right\}$ of $\mathbb{N}$ with $n^{\prime} \rightarrow \infty$, there is a subsequence $\left\{n^{\prime \prime}\right\}$ of $\left\{n^{\prime}\right\}$ with $n^{\prime \prime} \rightarrow \infty$ such that 25 holds. Hence

$$
E \sup _{x}\left|P_{\xi}\left(U_{n, \infty} \leq x \mid Z_{n}>0\right)-\Phi(x)\right| \rightarrow 0 .
$$

This gives 12 for $k=\infty$. The proof for $k \in \mathbb{N}^{*}$ is similar.

We now begin to prove 13 .

As we have proved that for each subsequence $\left\{n^{\prime}\right\}$ of $\mathbb{N}$, there is a subsequence $\left\{n^{\prime \prime}\right\}$ so that 24 holds, which implies: for a.e. $\xi$ and all $x \in \mathbb{R}$, as $n^{\prime \prime} \rightarrow \infty$,

$$
\left|P_{\xi}\left(U_{n^{\prime \prime}, \infty} \leq x \mid Z_{n^{\prime \prime}}>0\right)-\Phi(x)\right| \rightarrow 0 \text {. }
$$

It follows that for a.e. $\xi$ and all $x \in \mathbb{R}$,

$$
\left|P_{\xi}\left(U_{n^{\prime \prime}, \infty} \leq x, Z_{n^{\prime \prime}}>0\right)-P_{\xi}\left(Z_{n^{\prime \prime}}>0\right) \Phi(x)\right| \rightarrow 0
$$

So by the dominated convergence theorem, we see that for each $x \in \mathbb{R}$, as $n^{\prime \prime} \rightarrow \infty$,

$$
\left|P\left(U_{n^{\prime \prime}, \infty} \leq x, Z_{n^{\prime \prime}}>0\right)-P\left(Z_{n^{\prime \prime}}>0\right) \Phi(x)\right| \rightarrow 0
$$


and hence

$$
P\left(U_{n^{\prime \prime}, \infty} \leq x \mid Z_{n^{\prime \prime}}>0\right) \rightarrow \Phi(x) .
$$

By Dini's Theorem, it follows that

$$
\sup _{x}\left|P\left(U_{n^{\prime \prime}, \infty} \leq x \mid Z_{n^{\prime \prime}}>0\right)-\Phi(x)\right| \rightarrow 0 .
$$

Therefore we have proved that for each sequence $\left\{n^{\prime}\right\}$ of $\mathbb{N}$, there is a subsequence $\left\{n^{\prime \prime}\right\}$ of $\left\{n^{\prime}\right\}$ with $n^{\prime \prime} \rightarrow \infty$ such that 26 holds. Hence

$$
\sup _{x}\left|P\left(U_{n, \infty} \leq x \mid Z_{n}>0\right)-\Phi(x)\right| \rightarrow 0 .
$$

Thus the proof is completed.

\section{Proof of Theorem 2.2}

In this section, we consider the rate of convergence in the central limit theorem under a moment condition of order $2+\delta$, in proving Theorem 2.2 .

Notice that by the definition (4) of $m_{n}(a)$, we have

$$
m_{n}(a)=E_{\xi} X_{n, i}^{a} \text { if } a>0, m_{n}(a)=E_{\xi} X_{n, i}^{a} \mathbf{1}_{\left\{X_{n, i}>0\right\}} \text { if } a \leq 0,
$$

where $X_{n, i}$ is as in (2). For $a>0$, define

$$
R_{n}=\left[m_{0}(-a) \cdots m_{n-1}(-a)\right]^{-1} Z_{n}^{-a} \mathbf{1}_{\left\{Z_{n}>0\right\}}, \quad n \geq 0 .
$$

Lemma 4.1. $\left(R_{n}, \mathcal{F}_{n}\right)_{n \geq 0}$ is a supermartingale, where $\mathcal{F}_{n}$ were defined in (8).

Proof. Using the decomposition (2) of $Z_{n+1}$, we have

$$
\begin{aligned}
Z_{n+1}^{-a} \mathbf{1}_{\left\{Z_{n+1}>0\right\}} & =\left[\sum_{i=1}^{Z_{n}} X_{n, i}\right]^{-a} \mathbf{1}_{\left\{Z_{n}>0\right\}} \mathbf{1}_{\left\{Z_{n+1}>0\right\}} \\
& =Z_{n}^{-a}\left[\frac{1}{Z_{n}} \sum_{i=1}^{Z_{n}} X_{n, i} \mathbf{1}_{\left\{X_{n, i}\right\}>0}\right]^{-a} \mathbf{1}_{\left\{Z_{n}>0\right\}} \mathbf{1}_{\left\{Z_{n+1}>0\right\}} \\
& \leq Z_{n}^{-a} \frac{1}{Z_{n}} \sum_{i=1}^{Z_{n}}\left(X_{n, i} \mathbf{1}_{\left\{X_{n, i}\right\}>0}\right)^{-a} \mathbf{1}_{\left\{Z_{n}>0\right\}} \mathbf{1}_{\left\{Z_{n+1}>0\right\}}
\end{aligned}
$$

where the last inequality is due to the convexity property of the function $x^{-a}(a>$ $0)$.

Taking conditional expectation with respect to $\mathcal{F}_{n}$ and $P_{\xi}$ on both sides of the above inequality, we obtain that

$$
E_{\xi}\left(Z_{n+1}^{-a} \mathbf{1}_{\left\{Z_{n+1}>0\right\}} \mid \mathcal{F}_{n}\right) \leq Z_{n}^{-a} \mathbf{1}_{\left\{Z_{n}>0\right\}} m_{n}(-a),
$$

which gives the desired result. 
Since $Z_{0}=1$, by (28), we immediate obtain the following

Lemma 4.2. For $a>0$, we have

$$
E_{\xi} Z_{n}^{-a} \mathbf{1}_{\left\{Z_{n}>0\right\}} \leq m_{0}(-a) \cdots m_{n-1}(-a)
$$

If the environment sequence $\left\{\xi_{n}\right\}$ is independent and identically distributed, then

$$
E Z_{n}^{-a} \mathbf{1}_{\left\{Z_{n}>0\right\}} \leq\left(E m_{0}(-a)\right)^{n} .
$$

Now we give the proof of Theorem 2.2 .

Proof of Theorem 2.2. We shall only deal with the case $k=\infty$, as the case where $k \in \mathbb{N}^{*}$ can be treated similarly.

Consider the probability space $\left(\Gamma^{*} \times \Theta^{\mathbb{N}}, P^{*}\right)$ and define random variables $Z_{n}^{*}$ as in the proof of Theorem 2.1. By definition,

$$
u_{n}\left(Z_{n}^{*}, x\right)=P_{\xi}\left(\frac{1}{\sqrt{Z_{n}^{*}}} \sum_{j=1}^{Z_{n}^{*}} \frac{W_{\infty}(n, j)-1}{\Delta_{\infty}\left(\theta^{n} \xi\right)} \leq x\right) .
$$

By our hypothesis and the Berry-Esseen theorem (see e.g. Theorem 6 of (Petrov 1995, p.115)), we have

$$
\begin{aligned}
\left|u_{n}\left(Z_{n}^{*}, x\right)-\Phi(x)\right| & \leq \frac{C_{\delta}}{\left(Z_{n}^{*}\right)^{1+\frac{\delta}{2}}} \sum_{j=1}^{Z_{n}^{*}} E_{\xi}\left|\frac{W_{\infty}(n, j)-1}{\Delta_{\infty}\left(\theta^{n} \xi\right)}\right|^{2+\delta} \\
& =C_{\delta}\left(Z_{n}^{*}\right)^{-\frac{\delta}{2}} E_{\theta^{n} \xi}\left|\frac{W_{\infty}-1}{\Delta_{\infty}}\right|^{2+\delta}
\end{aligned}
$$

where $C_{\delta}$ is the Berry-Esseen constant. Using this evaluation, we can derive that

$$
\begin{aligned}
\left|P_{\xi}\left(U_{n, \infty} \leq x \mid Z_{n}>0\right)-\Phi(x)\right| & \leq E_{\xi}^{*}\left|u_{n}\left(Z_{n}^{*}, x\right)-\Phi(x)\right| \\
& \leq C_{\delta} E_{\xi}^{*}\left(Z_{n}^{*}\right)^{-\frac{\delta}{2}} E_{\theta^{n} \xi}\left|\frac{W_{\infty}-1}{\Delta_{\infty}}\right|^{2+\delta} .
\end{aligned}
$$

By the definition of $Z_{n}^{*}$, this implies that

$$
\begin{aligned}
\mid P_{\xi}\left(U_{n, \infty} \leq x, Z_{n}>0\right) & -P_{\xi}\left(Z_{n}>0\right) \Phi(x) \mid \\
& \leq C_{\delta} E_{\xi}\left(Z_{n}^{-\frac{\delta}{2}} I_{\left\{Z_{n}>0\right\}}\right) E_{\theta^{n} \xi}\left|\frac{W_{\infty}-1}{\Delta_{\infty}}\right|^{2+\delta} .
\end{aligned}
$$

Using (31) and the fact that the sequence $\left\{\xi_{n}\right\}$ is independent and identically distributed, we get

$$
\begin{aligned}
& \left|P\left(U_{n, \infty} \leq x, Z_{n}>0\right)-P\left(Z_{n}>0\right) \Phi(x)\right| \\
\leq & E\left|P_{\xi}\left(U_{n, \infty} \leq x, Z_{n}>0\right)-P_{\xi}\left(Z_{n}>0\right) \Phi(x)\right| \\
\leq & C_{\delta} E\left(Z_{n}^{-\frac{\delta}{2}} I_{\left\{Z_{n}>0\right\}}\right) E\left|\frac{W_{\infty}-1}{\Delta_{\infty}}\right|^{2+\delta} .
\end{aligned}
$$


Together with (30), we obtain that

$$
\left|P\left(U_{n, \infty} \leq x \mid Z_{n}>0\right)-\Phi(x)\right| \leq \frac{C_{\delta}\left(E m_{0}\left(-\frac{\delta}{2}\right)\right)^{n} E\left|\frac{W_{\infty}-1}{\Delta_{\infty}}\right|^{2+\delta}}{P\left(Z_{n}>0\right)} .
$$

Then the proof is completed.

\section{Acknowledgements}

The authors would like to thank the Editor and an anonymous referee for their comments and remarks. The research was the supported by the National Natural Science Foundation of China (Grant No. 10771021, 10871064 and 50907005), the Key Labor. of Comput. Stoch. Math., Univ. of Hunan (No. 09K026) and the Scientific Research Foundation for the Returned Overseas Chinese Scholars, Ministry of Education of China (Grant No. [2008] 890).

\section{References}

Athreya, K. B., Karlin, S., 1971a. On branching processes with random environments. I. Extinction probabilities. Ann. Math. Statist. 42, 1499-1520.

Athreya, K. B., Karlin, S., 1971b. Branching processes with random environments. II. Limit theorems. Ann. Math. Statist. 42, 1843-1858.

Athreya, K. B., Ney, P. E., 1972. Branching processes. Springer-Verlag, New York, die Grundlehren der mathematischen Wissenschaften, Band 196.

Bühler, W. J., 1969. Ein zentraler Grenzwertsatz für Verzweigungsprozesse. Z. Wahrscheinlichkeitstheorie und Verw. Gebiete 11, 139-141.

Grincevičjus, A. K., 1974. The continuity of the distribution of a certain sum of dependent variables that is connected with independent walks on lines. Teor. Verojatnost. i Primenen. 19, 163-168.

Guivarc'h, Y., Liu, Q., 2001. Propriétés asymptotiques des processus de branchement en environnement aléatoire. C. R. Acad. Sci. Paris Sér. I Math. 332 (4), 339-344.

Harris, T. E., 1963. The theory of branching processes. Die Grundlehren der Mathematischen Wissenschaften, Bd. 119. Springer-Verlag, Berlin.

Heyde, C. C., 1971. Some central limit analogues for supercritical GaltonWatson processes. J. Appl. Probability 8, 52-59.

Heyde, C. C., Brown, B. M., 1971. An invariance principle and some convergence rate results for branching processes. Z. Wahrscheinlichkeitstheorie und Verw. Gebiete 20, 271-278. 
Jagers, P., 1974. Galton-Watson processes in varying environments. J. Appl. Probability 11, 174-178.

Petrov, V. V., 1995. Limit theorems of probability theory. Vol. 4 of Oxford Studies in Probability. The Clarendon Press Oxford University Press, New York, sequences of independent random variables, Oxford Science Publications.

Smith, W. L., Wilkinson, W. E., 1969. On branching processes in random environments. Ann. Math. Statist. 40, 814-827.

Tanny, D., 1977. Limit theorems for branching processes in a random environment. Ann. Probability 5 (1), 100-116. 